\title{
Theoriegeschichte in systematischer Absicht
}

\author{
Wolfgang Schluchters »Grundlegungen der Soziologie« in der Diskussion \\ Hrsg. v. Hans-Peter Müller u. Steffen Sigmund
}

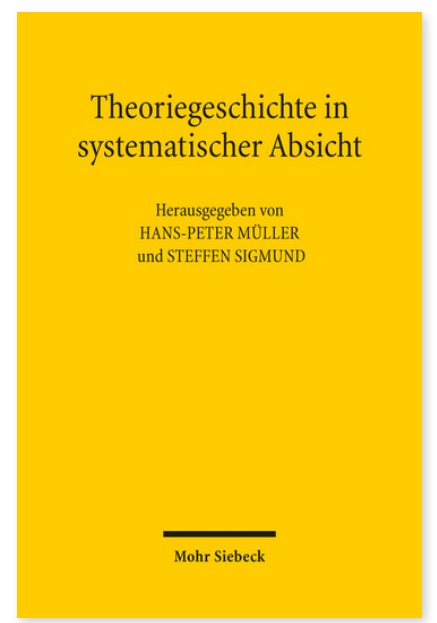

2017. VI, 316 Seiten.

ISBN 978-3-16-154436-1 DOI 10.1628/978-3-16-154436-1 eBook PDF 79,00€

ISBN 978-3-16-153621-2 fadengeheftete Broschur 79,00€
Wie kaum ein Zweiter hat Wolfgang Schluchter in seinem Grundlagenwerk Klassikerinterpretation mit Theoriebildung verknüpft. Was heißt »Theoriegeschichte in systematischer Absicht«? Wie sehen die Konturen dieses Forschungsprogramms aus? Welche Vorzüge, aber gegebenenfalls auch Grenzen weist dieses Programm auf? Das sind die zentralen Fragen, die im vorliegenden Band von namhaften Soziologen und Sozialtheoretikern diskutiert werden. Von der Auseinandersetzung mit den Klassikern der Gesellschaftstheorie wie Karl Marx, Max Weber, Emile Durkheim oder Niklas Luhmann führt diese Diskussion zur Frage nach der Bedeutung von historisch informierter, systematischer Theoriebildung in der Soziologie heute. Wie ist sie angesichts des heutigen vielfältigen Theorieangebots noch möglich? Mit einem Beitrag von Wolfgang Schluchter, in dem er eine mögliche Fortschreibung seiner Grundlegungen der Soziologie skizziert.

\section{Inhaltsübersicht}

Gert Albert: Das Forschungsprogramm von Vilfredo Pareto (Arbeitstitel) - Harald Bluhm: Die Konzeptualisierung der Handlungsmittel bei Karl Marx und deren Relevanz für Max Weber - Alessandro Cavalli: Die Präsenz der Absenz. Georg Simmel und Max Weber - Frank Ettrich: Das Forschungsprogramm von Karl Marx. Divergenz oder Konvergenz mit Max Weber - Hans-Peter Müller: Emile Durkheim in der französischen Soziologie - Armin Nassehi: Die Systemtheorie von Niklas Luhmann - Matthias Koenig: Die Vitalität des durkheimianischen Forschungsprogramms in der gegenwärtigen Soziologie Georg Kneer: Theorieexplikation und Theoriesynthese. Ein Kommentar zu Wolfgang Schluchters Grundsätzen einer strukturalistisch-individualistischen verstehenden Soziologie - Karl-Siegbert Rehberg: Max Webers kantianisierende Soziologie (Arbeitstitel) - Uwe Schimank: Der weite Weg zum common ground - vorbei an Ab- und Scheidewegen - Wolfgang Schluchter: Die Dualität von Struktur und Handlung. Zur Weiterentwicklung des Weberschen Forschungsprogramms Michae/ Schmid: Die systemtheoretische Wende - Talcott Parsons - Thomas Schwinn: Offene Fragen einer kantianisierenden Soziologie - Steffen Sigmund: Was heißt Theoriegeschichte in systematischer Absicht? - Rudolf Stichweh: System, Handlung, Kommunikation. Grundbegriffe der Sozialwissenschaften - Harald Wenzel: Die sprachtheoretische Wende - George H. Mead und Jürgen Habermas

Hans-Peter Müller Geboren 1951; Studium der Wirtschafts- und Sozialwissenschaften; 1981 Promotion; 1990 Habilitation; seit 1992 Professor für Allgemeine Soziologie an der Humboldt-Universität Berlin.

Steffen Sigmund Geboren 1961; Studium der Soziologie, Politische Wissenschaft, Geschichte, Volkswirtschaftslehre; 1997 Promotion; seit 2010 Professor für Empirische Makrosoziologie an der Ruprecht-Karls-Universität Heidelberg.

Jetzt bestellen:

https://mohrsiebeck.com/buch/theoriegeschichte-in-systematischer-absicht-9783161544361?no cache=1 order@mohrsiebeck.com

Telefon: +49 (0)7071-923-17

Telefax: +49 (0)7071-51104 